\title{
Exciton decay dynamics in individual carbon nanotubes at room temperature
}

\author{
Tobias Gokus, ${ }^{1, a)}$ Achim Hartschuh, ${ }^{1}$ Hayk Harutyunyan, ${ }^{2}$ Maria Allegrini, ${ }^{2}$ \\ Frank Hennrich, ${ }^{3}$ Manfred Kappes, ${ }^{3}$ Alexander A. Green, ${ }^{4}$ Mark C. Hersam, ${ }^{4}$ \\ Paulo T. Araújo, ${ }^{5}$ and Ado Jorio ${ }^{5}$ \\ ${ }^{1}$ Department Chemie und Biochemie, Ludwig-Maximilians-Universität München and CeNS, \\ 81377 München, Germany \\ ${ }^{2}$ Dipartimento di Fisica “E. Fermi," Universitá di Pisa and CNISM, Largo Pontecorvo 3, 56127 Pisa, Italy \\ ${ }^{3}$ Forschungszentrum Karlsruhe, Institut für Nanotechnologie, D-76021 Karlsruhe, Germany \\ ${ }^{4}$ Department of Materials Science and Engineering, Department of Chemistry, Northwestern University, \\ Evanston, Illinois 60208-3108, USA \\ ${ }_{5}^{5}$ Departamento de Física, Universidade Federal de Minas Gerais, Belo Horizonte, Minas Gerais, \\ 30123-970, Brazil
}

(Received 6 February 2008; accepted 2 April 2008; published online 18 April 2008)

\begin{abstract}
We studied the exciton decay dynamics of individual semiconducting single-walled carbon nanotubes at room temperature using time-resolved photoluminescence spectroscopy. The photoluminescence decay from nanotubes of the same $(n, m)$ type follows a single exponential decay function, however, with lifetimes varying between about 1 and 40 ps from nanotube to nanotube. A correlation between broad photoluminescence spectra and short lifetimes was found and explained by defects promoting both nonradiative decay and vibronic dephasing. (C) 2008 American Institute of Physics. [DOI: 10.1063/1.2913009]
\end{abstract}

Based on their exceptional optical properties, singlewalled carbon nanotubes (SWNT) will eventually play an important role as nanometer-scale building blocks for optoelectronics, nanoelectronics, and biosensing. ${ }^{1-3}$ Experimental and theoretical studies confirmed the identification of the photoluminescent state as being excitonic in nature with extremely large exciton binding energies. ${ }^{4,5}$ The excited state energies and dynamics of SWNT attracted particular scientific interest motivated by their unique one-dimensional structure combining intriguing optical and transport properties. To date, a large number of ensemble studies using pump-probe and time-resolved photoluminescence (PL) spectroscopy exist, reporting on mono- or multiexponential relaxation dynamics with decay times ranging from few to several tens of picoseconds for different sample materials. ${ }^{4,6,7}$ Temperature dependent PL measurements suggest that the excited state relaxation reflects a complex interplay between excitonic states of different parity that are optically bright or dark depending on their accessibility from the ground state. ${ }^{8,9}$ At present, excited state lifetimes are thought to be limited by fast transitions to these dark states but also by quenching related to defect related trap states. Transitions between excitonic states of different parity are expected to require symmetry breaking defects or environmental perturbations. ${ }^{10}$

Defects and environmental coupling are spatially localized by nature and a unique property of a given nanotube. As a result, ensemble measurements will always represent an averaging. Previous single nanotube PL measurements revealed a distribution of lifetimes for a single nanotube chirality $(6,4)$ ranging from sub 20 to $180 \mathrm{ps}$ with an average value of $57 \mathrm{ps}$ at $87 \mathrm{~K}$. ${ }^{11}$ These studies were limited to low temperatures because of the time resolution of the utilized system. In this paper, we report on the first time-resolved PL

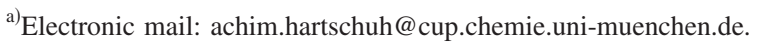

measurements of individual $(6,4)$ and $(6,5)$ SWNT at room temperature. PL transients extending over more than four orders of magnitude were found to be monoexponential with lifetimes ranging from about 1 to $40 \mathrm{ps}$.

Single nanotube measurements were performed using an inverted confocal microscope in combination with electronics for time-correlated single-photon counting. Laser excitation is provided by a femtosecond Ti:sapphire laser operating at $760 \mathrm{~nm}$ and a repetition rate of $76 \mathrm{MHz}$. A high numerical aperture objective $(\mathrm{NA}=1.3)$ provides maximum collection efficiency required for single nanotube detection. ${ }^{12}$ Spatially isolated individual nanotubes were obtained by spin coating a small volume of micelle-encapsulated SWNT onto a glass coverslip. The SWNT were enriched in the $(6,5)$ chirality through density gradient ultracentrifugation of CoMoCat material $^{13,14}$ and dialyzed into sodium cholate solution to remove any density gradient medium (i.e., iodixanol) remaining in the sample. Atomic force microscopy (AFM) measurements were carried out to verify low sample coverage of about $0.6 \mathrm{SWNT} / \mu \mathrm{m}^{2}$. Photoluminescent nanotubes were located by raster scanning the sample and simultaneous detection of the PL intensity between 800 and $1020 \mathrm{~nm}$ using either a fast avalanche photodiode (APD) after spectral filtering or a charge coupled device camera in combination with a spectrograph. In this spectral range chirality assignment based on the PL energy is unambiguous due to the small number of emissive nanotube structures. The instrument response function (IRF) of the system was independently measured by detecting the scattered pump laser light from the sample and had a full width at half maximum (FWHM) of 27 ps. To ensure optimum performance, the IRF was repeatedly recorded in between measurements. PL transients were fitted by exponential functions that were convoluted with the IRF. Due to the high signal to noise ratio achieved in our experiments and the reproducibility of the 

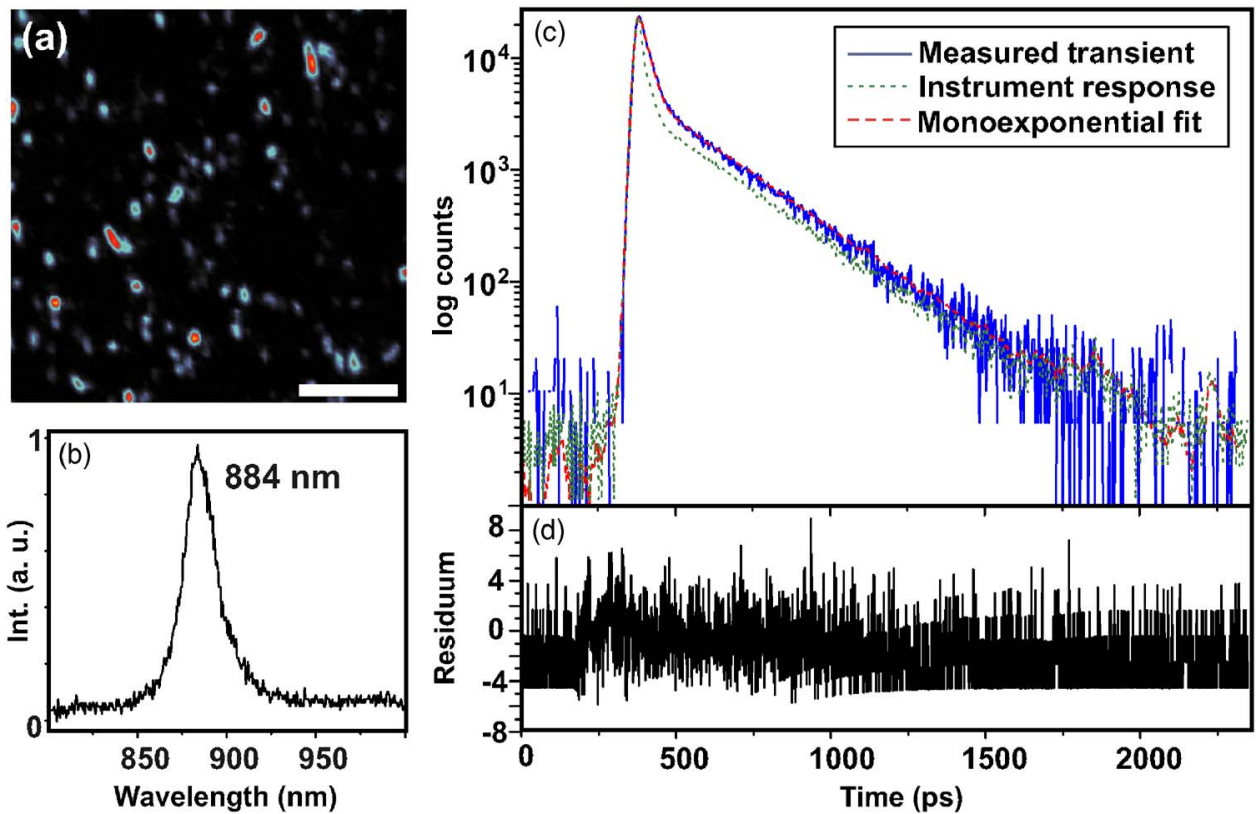

FIG. 1. (Color online) (a) Confocal PL image of SWNT on glass using a detection range of $800-1020 \mathrm{~nm}$ (scalebar $5 \mu \mathrm{m})$. The excitation density was $35 \times 10^{12}$ photons/ $\left(\right.$ pulse $\left.\mathrm{cm}^{2}\right)$. (b) PL spectrum detected for one of the nanotubes in (a). Based on the emission energy of $884 \mathrm{~nm}$ the nanotube chirality is identified as $(6,4)$ (Ref. 16). (c) Semilogarithmic plot of the PL transient (solid line) detected for the $(6,4)$ nanotube observed in (b) together with the independently recorded instrument response function (IRF, dotted line). A single-exponential fit function (dashed line) convoluted with the IRF describes the transient over four orders of magnitude clearly showing monoexponential decay dynamics with a lifetime $\tau=15 \pm 3 \mathrm{ps}$. The quality of the fit can be seen from the residuum of measured transient and singleexponential fit in (d). Note. the second slope results from the IRF and is observed for all measured transients.
IRF the time resolution of the setup is about $3 \mathrm{ps,} \mathrm{close} \mathrm{to}$ $10 \%$ of the FWHM of the IRF. ${ }^{15}$

A typical PL image of nanotubes emitting between 800 and $1020 \mathrm{~nm}$ is shown in Fig. 1(a). The length of some of the PL features clearly exceeds the diffraction limited resolution of $\approx 400 \mathrm{~nm}$ indicating the presence of long nanotubes in agreement with AFM data recorded for this sample. The nanotube density of $0.4 / \mu \mathrm{m}^{2}$ observed in the optical image is in agreement with the value determined from AFM measurements and demonstrates that most of the nanotubes on the substrate are in fact emissive in the accessible spectral range. Chirality specific optical detection is achieved by inserting a narrow bandpass filter centered at $880 \mathrm{~nm}$, the energy of the $E_{11}^{S}$ transition of the $(6,4)$ nanotube or at $980 \mathrm{~nm}$, the energy of the $(6,5)$ nanotube. ${ }^{16}$ Figure $1(\mathrm{~b})$ shows a typical PL spectrum recorded for one of the nanotubes seen in (a). The emission wavelength of $884 \mathrm{~nm}$ allows for a clear identification of the nanotube chirality as $(6,4)$.

Figure 2(c) shows the corresponding PL transient of the $(6,4)$ nanotube together with the measured IRF function. The dashed line is the monoexponential fit giving an exciton decay time of $\tau=15 \pm 3 \mathrm{ps}$. The uniform profile of the residuum between measured curve and fit shown below in Fig. 2(d) clearly demonstrates that the exciton dynamics at room temperature can be described by a single-exponential decay covering more than four orders of magnitude.

Transients measured for several hundred different $(6,4)$ nanotubes consistently exhibited monoexponential decay. However, the determined lifetimes showed a broad distribution ranging from about 1 to $40 \mathrm{ps}$. Three transients are presented in Fig. 2(a) featuring lifetimes of $\tau=4$, 18, and 36 ps. The histogram of lifetimes measured for 126 different $(6,4)$ nanotubes within the same sample is given in Fig. 2(b). The distribution is centered at about 11 ps with a width of 16 ps. While no literature data exists for $(6,4)$ nanotubes at room temperature, the average lifetime of $14 \mathrm{ps}$ is in general agreement with values reported for other nanotube chiralities. ${ }^{17}$ The corresponding distribution established for $(6,5)$ nanotubes within the same sample results in an average lifetime of 5 ps and a similar width. Remarkably, this value agrees very well with the 6 ps determined by pump-probe measurements for the same CoMoCat nanotube material allowing for a direct comparison of the results achieved by both techniques. ${ }^{6}$ On the other hand, ensemble measurements need to result in multiexponential decay profiles in which fitted lifetimes will reflect summation over decay times with different contributions. Extremely long lifetimes in the range of nanoseconds as reported in the literature have not been observed in our single nanotube measurements. ${ }^{7}$ In very few cases, less than $5 \%$ of the measured transients, better fitting
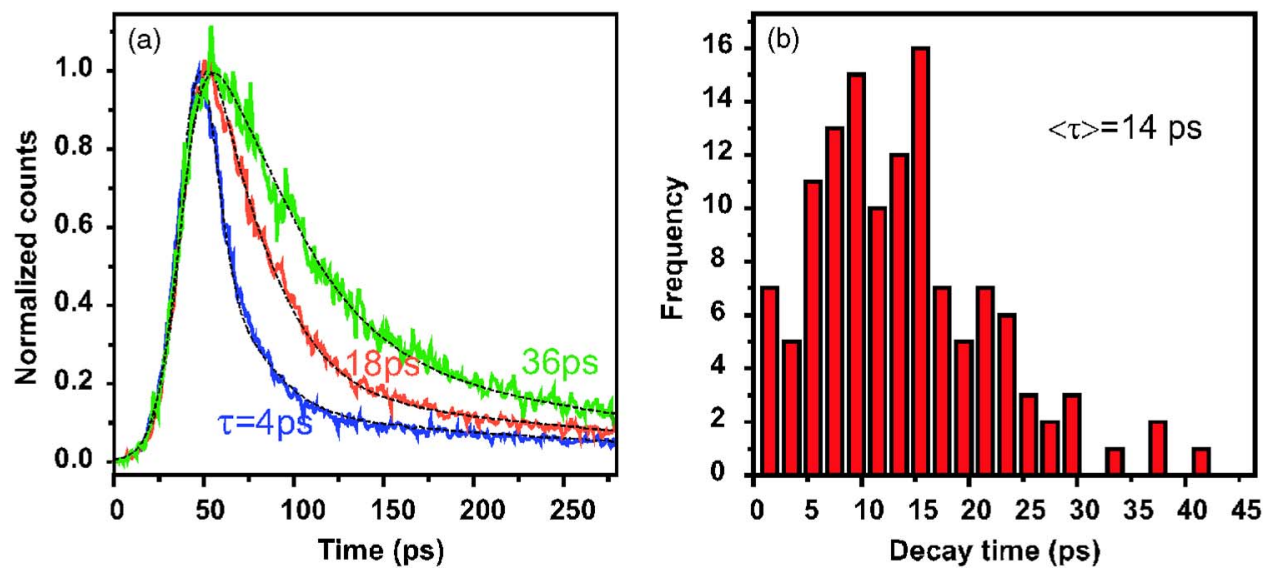

FIG. 2. (Color online) (a) PL transients recorded for three different single $(6,4)$ SWNT. Excited state decay times $\tau$ were obtained from monoexponential fits to the experimental data (solid lines) taking into account the measured instrument response. (b) Histogram of exciton decay times observed for 126 different $(6,4)$ nanotubes. The distribution is centered at $11 \mathrm{ps}$ with an average lifetime of $\langle\tau\rangle$ $=14 \mathrm{ps}$ and a width of $16 \mathrm{ps}$. 

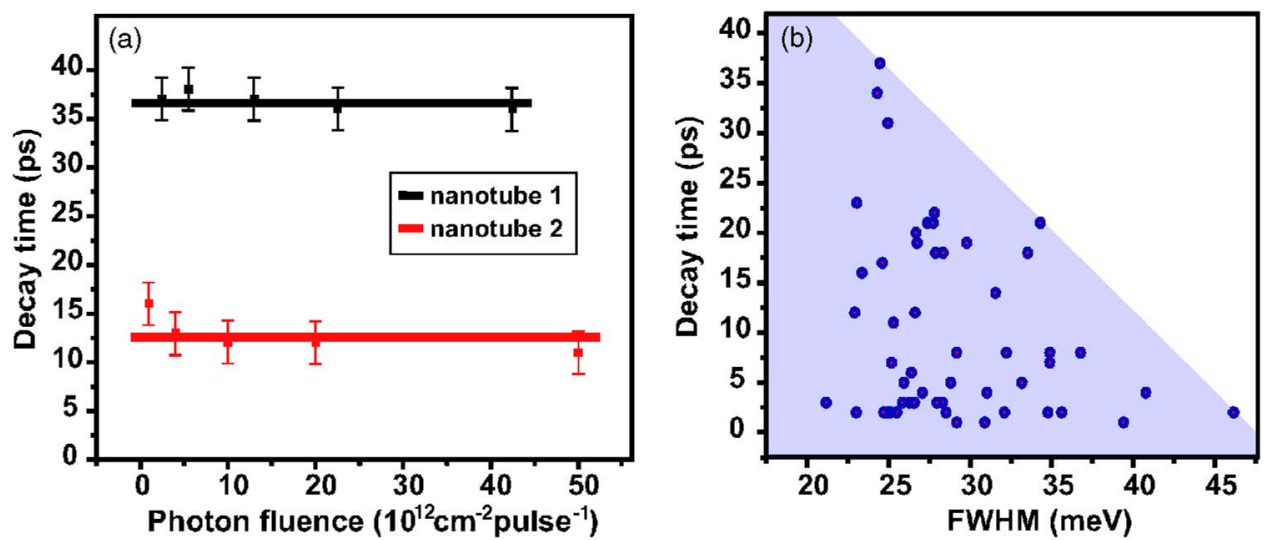

FIG. 3. (Color online) (a) Decay times determined for two different $(6,4)$ SWNT for increasing pump fluences. (b) Correlation between spectral width of detected $(6,4)$ nanotube spectra and lifetimes. Slow decay is observed for narrow linewidth. results could be achieved using multiple but similar decay times, probably because of spatial averaging along inhomogeneous nanotubes or the presence of a second nanotube within the detection area.

For high excitation densities exceeding $10^{14}$ photons / $\left(\mathrm{cm}^{2}\right.$ pulse $)$, exciton-exciton annihilation or Auger-recombination was observed to dominate the excited state dynamics. ${ }^{18}$ In the range of the low pump fluences used in this study $\left(P=2-50 \times 10^{12}\right.$ photons $/\left(\mathrm{cm}^{2}\right.$ pulse $)$ the measured transients resulted in constant lifetimes to within \pm 2 ps [Fig. 3(a)] ruling out that the lifetime variations reported here result from multiexciton effects. Indeed, for higher excitation densities around $10^{15}$ photons $/\left(\mathrm{cm}^{2}\right.$ pulse $)$ we continuously observed decreasing decay times. In few cases we observed irreversible photodegradation of the nanotubes at highest excitation densities leading to reduced PL intensities and lifetimes.

The emission energies of the observed $(6,4)$ nanotubes were found to vary by about $20 \mathrm{meV}$ (data not shown). Such variations have been reported in previous studies and can be assigned to heterogeneities in the dielectric environment. ${ }^{19-21}$ No correlation was found between the emission energy and the lifetime of the nanotubes. Long lifetimes, on the other hand, were mostly associated with narrow emission linewidth and short lifetimes with broad linewidth while no exclusive 1:1 correlation could be observed [Fig. 3(b)]. Homogeneous broadening of Lorentzian line shapes results from dephasing described by the dephasing time $T_{2}^{*}$ and the finite lifetime $\tau$ of the excited state leading to the linewidth $\Gamma$ $=1 / T_{2}^{*}+1 / \tau$. Since $\tau$ is in the range of several picoseconds, dephasing will be the dominating process controlling $\Gamma$. Dephasing times $T_{2}^{*}$ in the femtosecond range were recently predicted by theoretical calculations depending on the presence of structural defects. ${ }^{22}$ Very fast dephasing is predicted for certain types of defects that could also promote fast nonradiative decay.

In summary, we presented time-resolved PL measurements of a large number of single nanotubes at room temperature. In nearly all cases, exciton decay is found to be monoexponential with decay times varying between 1 and 40 ps. While average decay times are in excellent agreement with recent pump-probe data of the same nanotube material, future studies will need to clarify the origin of the lifetime variation.
We thank H. Qian and N. Hartmann for experimental assistance. Financial support by the DAAD, CAPES, and the DFG through Grant No. HA4405/3-1 and Nanosystem Initiative München (NIM) is gratefully acknowledged. This work was also funded by the U.S. National Science Foundation under Award Nos. EEC-0647560 and DMR-0706067.

${ }^{1}$ Topics in Applied Physics, edited by A. Jorio, M. S. Dresselhaus, and G. Dresselhaus (Springer, Berlin/Heidelberg, 2008), Vol. III.

${ }^{2}$ W. Lu and C. M. Lieber, Nat. Mater. 6, 841 (2007).

${ }^{3}$ P. W. Barone, B. Seunghyun, D. A. Heller, and M. S. Strano, Nat. Mater. 4, 86 (2004)

${ }^{4}$ F. Wang, G. Dukovic, L. E. Brus, and T. F. Heinz, Science 308, 838 (2005).

${ }^{5}$ J. Maultzsch, R. Pomraenke, S. Reich, E. Chang, D. Prezzi, A. Ruini, E. Molinari, M. S. Strano, C. Thomsen, and C. Lienau, Phys. Rev. B 72, 241402(R) (2005)

${ }^{6}$ Z. Zhu, J. Crochet, M. S. Arnold, M. C. Hersam, H. Ulbricht, D. Resasco, and T. Hertel, J. Micro/Nanolith. MEMS MOEMS 111, 3831 (2006).

${ }^{7}$ M. Jones, W. K. Metzger, T. J. McDonald, C. Engtrakul, R. J. Ellingson, G. Rumbles, and M. J. Heben, Nano Lett. 7, 300 (2007).

${ }^{8}$ I. B. Mortimer and R. J. A. Nicholas, Phys. Rev. Lett. 98, 027404 (2007).

${ }^{9}$ G. D. Scholes, S. Tretiak, T. J. McDonald, W. K. Metzger, C. Engtrakul, G. Grumbles, and M. J. Heben, J. Micro/Nanolith. MEMS MOEMS 111, 11139 (2007).

${ }^{10}$ V. Perebeinos, J. Tersoff, and P. Avouris, Nano Lett. 5, 2495 (2005).

${ }^{11}$ A. Hagen, M. Steiner, M. B. Raschke, C. Lienau, T. Hertel, H. Qian, A. J. Meixner, and A. Hartschuh, Phys. Rev. Lett. 95, 197401 (2005).

${ }^{12}$ L. Novotny and B. Hecht, Principles of Nano-optics (Cambridge University Press, Cambridge, 2006).

${ }^{13}$ M. S. Arnold, A. A. Green, J. F. Hulvat, S. I. Stupp, and M. C. Hersam, Nat. Nanotechnol. 1, 60 (2006).

${ }^{14}$ A. A. Green and C. Hersam, Mater. Today 10, 59 (2007).

${ }^{15}$ D. V. O'Connor and D. Phillips, Time Correlated Single Photon Counting (Academic, London, 1984).

${ }^{16}$ S. M. Bachilo, M. S. Strano, C. Kittrell, R. H. Hauge, R. Smalley, and R. B. Weisman, Science 298, 2361 (2002)

${ }^{17}$ T. Hertel, A. Hagen, V. Talalaev, K. Arnold, F. Hennrich, M. Kappes, S. Rosenthal, J. McBride, H. Ulbricht, and E. Flahaut, Nano Lett. 5, 511 (2005).

${ }^{18}$ F. Wang, G. Dukovic, E. Knoesel, L. E. Brus, and T. F. Heinz, Phys. Rev. B 70, 241403 (2004).

${ }^{19}$ H. Htoon, M. J. O'Connell, P. J. Cox, S. K. Doorn, and V. I. Klimov, Phys. Rev. Lett. 93, 027401 (2004).

${ }^{20}$ A. Hartschuh, H. N. Pedrosa, L. Novotny, and T. D. Krauss, Science 301, 1354 (2003).

${ }^{21}$ A. Hartschuh, H. N. Pedrosa, J. Peterson, L. Huang, P. Anger, H. Qian, M. Steiner, A. J. Meixner, L. Novotny, and T. D. Krauss, ChemPhysChem 6, 577 (2005).

${ }^{22}$ B. F. Habenicht, H. Kamisaka, K. Yamashita, and O. V. Prezhdo, Nano Lett. 7, 3260 (2007). 